\title{
Violencia obstétrica: Una forma invisibilizada de la violencia contra las mujeres en Costa Rica
}

Trabajo Resultados de Investigación

Keylor Robles-Murillo

\section{Acercamiento a la violencia obstétrica}

La violencia contra las mujeres basada en género, se ha convertido en un fenómeno social que se caracteriza por su condición estructural, prolongación sociohistórica y legitimación en las relaciones de poder basadas en el sistema patriarcal y capitalista. Por esta razón, dicha temática se consolida como un objeto de estudio para las Ciencias Sociales, el cual puede ser abordado desde diversas aristas teóricas, epistemológicas y ontológicas que intentan comprender y problematizar manifestaciones concretas que se reproducen en los escenarios de la vida cotidiana.

Hidalgo y Rodríguez (2014) manifiestan que las mujeres habitan en un contexto en común, donde han sufrido una historia de dominio, explotación y opresión, coexistiendo en una realidad discriminatoria y en una sociedad dividida según el sexo, el poder adquisitivo, el nivel económico, el grado de escolaridad, la edad, la nacionalidad y la postura política.

Para analizar críticamente este fenómeno, y a su vez, generar rupturas con el orden patriarcal, se debe partir de los planteamientos de proyectos éticos-políticos ${ }^{8}$ que se orienten a la

7 Trabajador Social, Universidad de Costa Rica, Sede Occidente (UCR SO), Provincia de Alajuela, San Ramón, Costa Rica. Estudiante de Enseñanza del Castellano y Literatura, UCR SO. robleskeylor@gmail.com

8 Durán (2012) señala que el papel fundamental de la ética "como capacidad creadora del profesional [...] se expresa en la orientación de su práctica en referencia a la construcción de valores tales como la libertad, la justicia, la igualdad, la emancipación" (p. 135). 
transformación societal y emancipatoria. Esto implica comprender y visibilizar mediante procesos investigativos la opresión a la que se ven sometida las mujeres; generando una desigualdad estructural. Pelletier (2014) afirma que esta categoría conceptual incorpora particularidades históricas y sociales, reflejando situaciones de exclusión social sistemáticas.

Específicamente, en esta oportunidad, se decide profundizar en el análisis de la violencia obstétrica, enmarcada dentro de las múltiples formas de violencia contra las mujeres. Para este fin, se delimita como espacio territorial y escenario investigativo el contexto económico, social, político y cultural de Costa Rica durante el período del 2015 al 2016.

\section{Definiendo el proceso a seguir}

En primera instancia, se torna pertinente enfatizar que el proceso descrito, se define como una investigación de enfoque cualitativo. Según Creswell (citado por Vasilachis, 2006) este tipo de investigación se caracteriza por ser un proceso de indagación basado en distintas metodologías, las cuales abordan un "problema humano" mediante una construcción compleja y holística que se basa en palabras y perspectivas de las personas informantes. Es decir, toma en cuenta que es un proceso dialéctico en donde la deconstrucción y reconstrucción permanece constante. Lo anterior, resultó fundamental durante el trabajo, pues las experiencias personales fueron pilares al abordar la violencia obstétrica.

Seguidamente, en lo que respecta a los métodos de investigación empleados, se debe aludir al método biográfico. De acuerdo con García, Gil y Rodríguez (1996) este pretende mostrar el testimonio de una persona, recopilando los acontecimientos y valoraciones subjetivas que dicha persona hace de su propia existencia. Cabe agregar que la técnica definida para llevar a cabo su proceso metodológico fue la entrevista a profundidad. Esta se puede definir como la reconstrucción de trayectorias pasadas y el estudio de representaciones sociales personalizadas; refiriéndose a temas complejos y difíciles (Mejía, 2004).

Cabe añadir que las razones principales por las cuales se desarrollaron entrevistas a profundidad, corresponde a que, en primer lugar, permite reconstruir las expresiones de la violencia obstétrica desde la experiencia 
genérica de ellas, pArtiendo de los relatos comentados desde sus subjetividades, incluyendo sentires y pensares. Además, se pueden abordar las representaciones sociales que han construido las mujeres participantes sobre el parto y la maternidad, las cuales influyen directamente en su posición ante la violencia obstétrica.

Referente a la población seleccionada, es importante hacer alusión a que la delimitación de esta, tuvo el objetivo de evidenciar la existencia de factores múltiples de opresión. Maffia (2007) asegura que las mujeres no solo son discriminadas sino también segregadas. Agregando a lo expuesto por la autora se considera que todas las mujeres son discriminadas por la categoría sexo-género; sin embargo, esta se agrava cuando converge con otra serie de factores.

Por esta razón, se intentó analizar la influencia de otras categorías dentro de las experiencias de violencia obstétrica de las participantes. En este caso se abordaron los factores: etario, condición migratorio y centro-periferia. Se puede agregar que la inclusión de estas características, se originó como un esfuerzo de investigación innovador, al aportar elementos de discusión y análisis que no habían sido abordados previamente9.

En el primer caso, la mujer participante tenía dieciocho años de edad y residía en la Región de Occidente de Costa Rica, cuando se ejecutó el trabajo de campo. Cabe enfatizar que la entrevistada era menor de edad al momento del alumbramiento. En la experiencia vivencial de ella, se desea analizar cómo se agrava la violencia obstétrica cuando entra en juego el factor etario. De acuerdo con Jager, López y Vargas (2005) este tipo de discriminación se fundamenta en la edad de las personas, es decir, en un número que expone la cantidad de años vividos.

El segundo relato fue expuesto por una participante de cuarenta años que habitaba en la Región Brunca del territorio nacional. En la situación de la mujer descrita, se posiciona la discriminación que surge entre el proceso

9 Es trascendental destacar que al momento de realizar el proceso investigativo, solamente se había desarrollado una producción académica sobre la violencia obstétrica en Costa Rica; titulada "La violencia obstétrica: propuesta conceptual a partir de la experiencia costarricense", la cual fue llevada a cabo por Gabriela Arguedas (2014). 
centro-periferia. Se torna pertinente añadir que a pesar que la delimitación se establece de manera cartográfica (centro: Gran Área Metropolitana / periferia: Zona Sur), este análisis no puede ser concebido solamente en términos cartográficos, es decir, debe trascender la localización contextual geográfica.

La participante restante fue una mujer migrante de veinticinco años residente del Gran Área Metropolitana desde el año 2005. En el caso específico de ella, se desea estudiar la discriminación ocasionada por la nacionalidad. Costa Rica, al igual que otros países latinoamericanos, ha experimentado flujos migratorios desde el siglo XIX, dando como resultado un país multiétnico y pluricultural. Sin embargo, siguen predominando diferentes perspectivas que se gestan en la construcción de la estereotipa alrededor de cada nacionalidad. Lamentablemente, la nacionalidad nicaragüense es una de las más inferiorizadas debido a que se considera como una minoría étnica, es decir, ciudadanos y ciudadanas de tercera clase ${ }^{10}$.

Finalmente, el análisis de resultados se llevó a cabo mediante una triangulación hermenéutica que consiste en un proceso en donde se reúne y se cruza dialécticamente la información relacionada con el objeto de estudio, surgida mediante el proceso investigativo (Cisterna, 2005). En este caso se trianguló la categoría sexo-género con diversas subcategorías, incluyendo las historias de vida, los aportes teórico-metodológicos y enfoque del investigador.

\section{Visibilización de la violencia obstétrica a partir del proceso investigativo}

En lo que refiere a los resultados obtenidos, estos se pueden colocar en tres grandes aspectos identificados a partir del trabajo de campo encauzado. Primeramente, la interiorización cultural de la maternidad como "plan de vida" impuesto a todas las mujeres. Aunado a esto, la naturalización de las maternidades forzosas por parte del Estado. En un tercer momento, se analiza la atención denigrante que se genera desde esquemas androcéntricos predominante en profesionales del servicio médico.

\footnotetext{
${ }^{10}$ Para ampliar este tema, veáse "Estudio exploratorio y binacional (Nicaragua y Costa Rica), sobre la incidencia de la migración en las mujeres” (Galo, Patiño y Solís, 2008).
} 
En primera instancia, respecto a la interiorización de la maternidad, una de las entrevistadas comentaba sobre el "sueño de ser madre" desde edades temprana. Afirmando lo siguiente: "desde niña crecí con el sueño de ser madre al igual que todas mis compañeras, yo creo que todas las mujeres nacemos con ese instinto materno, siempre nos preocupamos por todo el mundo; así soy yo, primero pienso en toda la demás gente y por último en mî" (Entrevistada \#1, 15 años, comunicación personal, 23 de agosto de 2015). Este elemento se repitió en las tres entrevistas realizadas a la población participante.

Agregando a lo anterior, se presenta una naturalización de las maternidades como imposición cultural producto del patriarcado. Esta perspectiva naturalizadora se reproduce en discursos emitidos por profesionales en ciencias médicas, similares al comentado por una de las sujetas participantes: "el doctor me gritaba: a usted nadie la mandó a abrir las piernas, así que aguante. Usted quería ser madre, entonces siga pujando y aprenda a ser mujer" (Entrevistada \#2, 40 años, comunicación personal, 13 de mayo de 2015). Esto evidencia que el sistema de salud es reproductor de prejuicios machistas y misóginos.

En tercer lugar, se refleja la atención denigrante que se les brinda a las mujeres migrantes. Esto se comenta en el relato de la entrevistada: "una enfermera me dijo que no me quejara y que me acostumbrara al sistema de salud de aquí [Costa Rica], y que si no me gustaba me podía devolver a Nicaragua" (Entrevistada \#3, 25 años, 4 de setiembre de 2015). La xenofobia en el sector salud en Costa Rica es un reflejo de la percepción de las personas nicaragüenses migrantes que dificulta ejercer, y ser concebidas, como ciudadanía.

\section{Discutiendo sobre violencia obstétrica}

Facio (1992) sostiene que las mujeres de todas las clases, razas, etnias, edades, opciones sexuales, entre otros factores, son oprimidas en razón de su sexo. Tal y como lo afirma esta autora, las manifestaciones de violencia contra la mujer basadas en género, se agravan cuando se interrelacionan con otros factores, es decir, no son las mismas realidades que enfrenta una mujer migrante que trabaja en el sector agropecuario, en comparación a una mujer que desempeña un cargo como ministra. 
Continuando con la descripción del sistema patriarcal, al cual se hace alusión en el párrafo anterior, Lerner (1986) afirma que "es una creación histórica elaborada por hombres y mujeres en un proceso que tardó casi 2.500 años en completarse. La primera forma de patriarcado apareció en el estado arcaico. La unidad básica de su organización era la familia patriarcal, que expresaba y generaba constantemente sus normas y valores" ( $p$. 311). Lo anterior revela que el sistema patriarcal, desde su consolidación, ha sido un sistema que se ha encargado de invisibilizar y oprimir a la mujer desde su inicio, resaltando la supuesta superioridad del hombre.

Dicho sistema legitima como prioritario todo aquello que se relacione con las características masculinizadas, y, a su vez, genera una acción inversa con lo feminizado socialmente, ya que se establece una relación de subordinación que predomina en todos los espacios de la vida cotidiana. Cabe enfatizar que, dentro de este Artículo, se afirma la presencia de la violencia contra la mujer basada en género, y no violencia de género, como en ocasiones se denomina, pues como lo señala Carcedo (2013) la violencia contra las mujeres corresponde a un problema de poder, suscitado por la desigualdad de poder entre los dos géneros (masculino y femenino). Si bien es cierto, el género debe incluirse como categoría analítica perteneciente a la teoría feminista, no puede ser empleado para homologar la violencia que sufren las mujeres cotidianamente con la violencia contra los hombres. Pues, la primera es histórica, estructural y sistemática, resultado del sistema patriarcal y las relaciones de poder (hombres=dominadores/mujeres=subordinadas) que se establecen.

De igual manera, se debe reiterar que, dentro de este texto, se concibe al patriarcado y al capitalismo como un sistema dual e intrínseco entre sí. De acuerdo con Izquierdo (1998), el capitalismo se entiende como un conjunto de relaciones sociales entre los sujetos enmarcados dentro de relaciones de explotación económica, cuya finalidad es la acumulación de capital. Por esta razón, se fundamenta en la separación entre trabajo y capital, en donde la burguesía controla los medios de producción, y la clase trabajadora modifican estas materias primas con el objetivo de producir mercancías que se inserten en la égida económica capitalista y neoliberal. Incluso la capacidad de trabajo, o fuerza de trabajo, de las personas se convierte en otra mercancía más. 
En este contexto, hablar de un sistema patriarcal-capitalista se fundamenta en la relación entre ambas formas de desigualdad. Como dice HArtmann (1980), no hay un capitalismo "puro", así como tampoco existe un patriarcado "puro", ya que los dos garantizan su coexistencia necesaria. Sumado a esto, Izquierdo (1998) enfatiza que no se puede afirmar que la sociedad es completamente capitalista o patriarcal, es decir, de manera segregada, ya que las formas de comportamiento se supeditan y son posible por el vínculo existente entre las dos estructuras opresoras. Incluso, manifiesta, con fines de ubicarles sociohistórico y concretamente, que se podría considerar que el fundamento del capitalismo se encuentra en el patriarcado; esto debido al momento de origen de ambos.

Para proseguir con el análisis, se percibe fundamental debatir específicamente sobre la violencia obstétrica, enmarcada dentro de las diversas manifestaciones de la violencia ejercida contra las mujeres en un sistema patriarcal y capitalista. Esto, con el objetivo de conceptualizar y, a su vez, visibilizar un fenómeno que suele pasarse desapercibido en las discusiones generadas sobre esta problemática estructural.

Continuando con esto, se debe mencionar que, a lo largo de las últimas cuatro décadas, algunas de las manifestaciones de la violencia contra la mujer han enfrentado un proceso continuo de desnaturalización, donde el objetivo corresponde a la búsqueda por erradicar totalmente dicha expresión específica. La mayoría de actoras sociales, ya sean individuales o colectivas, dentro del movimiento feminista se enfocaron esencialmente en la conquista de luchas sociales conexas con la participación política y la liberación femenina de diferentes formas de opresión cotidiana.

El ámbito de la atención del embarazo y todo lo afín con este: embarazo, parto, puerperio y lactancia, es solo uno de los escenarios invisibilizados en donde se ejemplifica la violencia ejercida contra las mujeres (Casas, Penagos y Pintado, 2015). No obstante, uno de los aspectos más relevantes de esta muestra de agresión es la naturalización presente dentro de los discursos y de las acciones cometidas por quienes participan en este proceso.

En lo que respecta a la visibilización de este fenómeno social, se debe manifestar que se caracteriza por su carácter reciente en relación a otras 
formas de violencia contra las mujeres, tales como la violencia física, sexual y psicológica. De forma concreta, este tema se empezó a abordar en el año 2004 en Argentina. Sin embargo, el término violencia obstétrica fue empleado por primera vez tres años después en Venezuela, siendo acuñado en la "Ley Orgánica sobre el Derecho de las Mujeres a una Vida Libre de Violencia”, aprobada en marzo del 2007. El objeto de esta ley se define en el siguiente Artículo:

La presente Ley tiene por objeto garantizar y promover el derecho de las mujeres a una vida libre de violencia, creando condiciones para prevenir, atender, sancionar y erradicar la violencia contra las mujeres en cualquiera de sus manifestaciones y ámbitos, impulsando cambios en los patrones socioculturales que sostienen la desigualdad de género y las relaciones de poder sobre las mujeres, para favorecer la construcción de una sociedad justa democrática, participativa, paritaria y protagónica (Artículo 1, Ley Orgánica sobre el Derecho de las Mujeres a una Vida Libre de Violencia, 2007).

Seguidamente, se debe hacer referencia a la conceptualización de este fenómeno. En el caso de Arguedas (2014), ella define la violencia obstétrica como "un conjunto de prácticas que degrada, intimida y oprime a las mujeres y a las niñas en el ámbito de la atención en salud reproductiva y, de manera mucho más intensa, en el período del embarazo, parto y postparto" (p.147). Asimismo, incorpora la categoría de "poder obstétrico" incluyendo los aportes teóricos de Foucault (citado por Arguedas 2014), en donde se afirma que "el poder obstétrico constituye una forma de poder disciplinario [...] que produce cuerpos sexuados y dóciles" (p.147), lo cual evidencia la forma en como el sistema patriarcal se encarga de intentar dominar el cuerpo de la mujer, realizando un proceso de cosificación y transformación en seres dóciles.

En el caso de Aragón (2013), ella agrega que la violencia obstétrica se presenta cuando se genera un proceso de apropiación del cuerpo y procesos reproductivos de las mujeres por prestadores de salud, dando como resultado tratos jerárquicos deshumanizadores y un abuso de medicalización y patologización de los procesos naturales. Lo anterior provoca pérdida de autonomía y capacidad para decidir libremente sobre sus cuerpos y sexualidad impactando negativamente en la calidad de vida de las mujeres. 
Para lograr una mejor comprensión de la violencia obstétrica se decide incluir la división de modalidades propuesta por Paolini (2011), quien clasifica esta expresión de violencia en: a) violencia obstétrica física y b) violencia obstétrica psíquica. Sobre esto, se debe aclarar que la autora coloca dos tipos de expresiones con características; empero, no pueden ser concebidas de manera aislada o excluyente. Pues en muchas de las situaciones, se presentan ambos casos.

En este texto, con fines de delimitación, se enfoca en el primer tipo de violencia obstétrica, el cual hace referencia a "prácticas invasivas y suministro de medicación que no estén justificados por el estado de salud de la parturienta o de la persona por nacer, además no respetar los tiempos ni las posibilidades del parto biológico" (Paolini, 2011, p.271), es decir, son aquellas acciones físicas cometidas en detrimento de la mujer y/o el niño o la niña. Aunado a esto, se debe incluir la realización de cesáreas innecesarias y otras praxis médicas peligrosas como la maniobra de Kristeller, la cual consiste en una:

Maniobra practicada durante el parto que consiste en presionar en la parte superior del útero durante las contracciones de la mujer. Se practica usualmente cuando el proceso de parto parece durar demasiado. Tiene potenciales efectos peligrosos como la rotura uterina, daños en el perineo, hematomas y contusiones en el abdomen de la mujer y el cuerpo del/la bebé (Sánchez, 2015, p. 111).

Igualmente, se puede mencionar la episiotomía, la cual consiste en una intervención quirúrgica en el perineo y paredes vaginales practicada durante el parto (Sánchez, 2015). El procedimiento descrito ha generado gran cantidad de debates con posiciones a favor y en contra, pues los colectivos de mujeres a favor del parto natural, afirman que la ejecución de esta práctica se realiza por "comodidad" del personal médico que interviene el proceso, transformando a las mujeres en objetos medicalizados.

En el caso de las mujeres entrevistadas, dos de ellas afirmaron que durante el alumbramiento, les realizaron una episiotomía sin previa consulta. Incluso una participante manifestó que esto le ocasionó problemas para realizar necesidades fisiológicas, y además le provocó impedimentos para trasladarse, pues la movilidad era sumamente reducida. 
Del mismo modo, se torna pertinente incluir los aportes de las médicas González y Ortíz (2005), quienes llevaron a cabo una indagación histórica, la cual contempla las transformaciones culturales más significativas que se han experimentado en relación con las prácticas y las poses de parto; lo que evidencia la influencia patriarcal:

Cuando Cristóbal Colón llegó a América [...] la posición vertical seguía siendo la dominante [...]. En el siglo XVII se produjo la muerte de María de Medecis durante el parto y como consecuencia la partera real francesa fue sustituida por Julien Clement, primer cirujano partero de la historia, quien introdujo la posición horizontal para beneficio del obstetra [...] (p.2).

En la actualidad, el parto que se efectúa se denomina parto tecnocrático, según Davis-Floyd (citado por Sánchez, 2015) esta modalidad desencadena el escenario donde se presenta la violencia obstétrica. Se torna pertinente destacar que dentro del presente texto se denomina parto hegemónico, pues se aplica la definición del concepto de hegemonía propuesto por Tasies (2015), quien alude que "una de las características principales de la hegemonía, expresa y materializa la apropiación de los mecanismos de dirección política de la sociedad" (p.8). Lo indicado previamente, refleja el poder político ejercido por la hegemonía para crear una acción que se sobrepone ante las demás. En este caso se direcciona la sociedad ante los mandatos sociales derivados del patriarcado, tomando como fin las particularidades que lo justifican.

Por otra parte, se evidencia un deterioro en los espacios destinados para las salas de parto, y otros sitios que son ocupados por las mujeres durante el alumbramiento y el período posterior. Sumado a esto, se evidencia en la escasez de personal profesional capacitado para situaciones obstétricas, pues se realiza una escasa apertura de plazas administrativas-profesionales para la contratación de especialistas en este campo. Según el informe publicado por la Defensoría de los Habitantes (2016) algunas falencias de las problemáticas identificadas son las siguientes:

Falta de especialistas en ginecología, neonatología, enfermería obstétrica u otras, falta de equipos (monitores fetales o monitores dúplex), poca disposición de camas o camas de expulsión en mal estado; falta 
de cortinas o biombos que garanticen el derecho a la intimidad, problemas de hacinamiento que producen que las mujeres se encuentren en los pasillos de las salas de maternidad lo que se presta para justificar la violencia del derecho al acompañamiento; situaciones de maltrato, regaños y, humillaciones a las mujeres durante el parto; además de la denegación de la atención prenatal a mujeres migrantes, lo que afecta la atención integral (p.6).

Además, en el documento se manifiesta que de los once centros de médicos en donde se encontraron estas problemáticas, cinco se ubican en la Región Brunca o Zona Sur del país: Hospital Fernando Escalante Pradilla, Hospital Tomas Casas Casajús, Hospital de San Vito, Hospital de Ciudad Neilly y Hospital de Golfito. Lo cual reitera que las condiciones del sistema de salud no son similares a los centros médicos que se ubican en el Gran Área Metropolitana; por tanto, se genera un detrimento en el servicio que se brinda. La mujer participante expresó lo siguiente: "Yo solamente soy otro de los casos de negligencia que han pasado en ese hospital, de hecho, yo escuché que ese hospital ha sido demandado varias veces por los malos tratos y por negligencias de todo tipo" (Entrevistada \#2, 40 años, comunicación personal, 13 de mayo de 2015).

Por último, dentro de la indagación realizada se evidenció una alternativa que ha surgido ante el parto hegemónico, vinculada directamente con el ejercicio del parto humanizado. Según Aragón (2013), el parto humanizado o natural se fundamenta en la valoración del "mundo afectivo emocional de las personas, la consideración de los deseos y necesidades de sus protagonistas: madre, padre, hija o hijo y la libertad de las mujeres o las parejas para tomar decisiones sobre dónde, cómo y con quién parir" (p.2). Esta alternativa intenta respetar la autonomía sobre los derechos sexuales y reproductivos de las mujeres, la cual ha sido arrebatada por el sistema patriarcal desde su consolidación.

\section{Conclusiones}

A modo de conclusión, se afirma que la violencia obstétrica, al igual que todas las manifestaciones de la violencia, se deben erradicar por completo. Por esta razón, es importante visibilizar dicha problemática con el objetivo de provocar cuestionamientos por parte de las mujeres que han 
experimentado estas situaciones para alcanzar rupturas que conlleven a la transformación societal.

Asimismo, se enfatiza que este tema debe analizarse como una problemática estructural, que se enmarca y responde a la lógica del sistema capitalista y patriarcal hegemónico, en donde las mujeres han sido oprimidas históricamente, y siguen siendo colocadas socialmente como ciudadanas de segunda categoría. Además, este sistema dual es el mismo que permite la reproducción de este fenómeno social dentro de los centros médicos públicos.

Al mismo tiempo, se divisa de carácter primordial generar discusiones sobre la problemática de la violencia obstétrica, las cuales se engloben dentro del marco de los derechos humanos, incluyendo la teoría feminista, en donde se perciba a las mujeres como sujetas dueñas de sus cuerpos. De igual forma, se reflexiona que los movimientos sociales y, especialmente, el movimiento feminista se consolida en un espacio desde el cual se puede visibilizar la problemática; por lo que se torna fundamental la incidencia de este movimiento en discusiones sobre dicha manifestación de la violencia contra la mujer.

Aunado a esto, se contempla ineludible impulsar iniciativas legales cuya finalidad corresponda a sancionar la violencia obstétrica; cabe mencionar que estas guías legislativas se deben realizar tomando en cuenta la voz de cada una de las mujeres que han sufrido violencia obstétrica, sin victimizarlas ni convertirlas en sujetas pasivas. A su vez, se debe exigir atención médica humanizada y de calidad; que garantice el cumplimiento a cabalidad de sus derechos sexuales y reproductivos, sin sesgos religiosos, políticos, ni moralistas.

Por último, en el caso de la profesión de Trabajo Social, y otras Ciencias Sociales, se deben encauzar intervenciones desde las diferentes dimensiones de atención, facilitando procesos individuales y grupales, en los cuales se aborden las manifestaciones de este fenómeno social, enmarcado dentro del sistema patriarcal, con el objetivo de brindar herramientas que permitan desarrollar un proceso de empoderamiento en las mujeres que han experimentado estas situaciones, y así avanzar en la eliminación de las formas de violencia que sufren las mujeres cotidianamente. 


\section{Referencias bibliográficas}

Aragón, M. (2013). Violencia obstétrica vs. Parto respetado. Apuntes para un debate urgente a través del texto de la Ley Nacional. Disponible en: http://cdsa.aacademica.org/000-038/741.pdf.

Arguedas, G. (2014). La violencia obstétrica: propuesta conceptual a partir de la experiencia costarricense. Cuadernos Intercambio sobre Centroamérica y el Caribe, 11(1), 145-169.

Carcedo, A. (2013). Violencia contra las mujeres: Un problema de poder. San José: CEFEMINA.

Casas, M.; Penagos, J. y Pintado, S. (2015). Síndrome de desgaste profesional en médicos y percepción de la violencia obstétrica. Ginecología y Obstetricia de México, 83, 173-178.

Cisterna, F. (2005). Categorización y triangulación como procesos de validación del conocimiento en investigación cualitativa. Theoria, 1(14), 61-71.

Defensoría de los Habitantes. (2016). Expediente Legislativo No19.537. San José: Costa Rica.

Durán, S. (2012). El proyecto ético-político del Trabajo Social: Aportes para la construcción de un proyecto profesional crítico desde la intervención en procesos de salud-enfermedad mental. Documentos de Trabajo Social, 51(22), 121-139.

Facio, A. (1992). Cuando el género suena cambios trae: Metodología para el análisis de género del fenómeno legal. San José: ILANUD.

Galo, C.; Patiño, M. y Solís, A. (2007). Estudio exploratorio y binacional (Nicaragua y Costa Rica), sobre la incidencia de la migración en las mujeres. San José: UNFPA.

García, E.; Gil, J. y Rodríguez, G. (1996). Metodología de la Investigación Cualitativa. Granada: Ediciones Aljibe.

González, E. y Ortíz, M. (2005). Posiciones maternas durante el parto. Alternativas a la posición ginecológica. Revista de la Facultad de Ciencias de la Salud, 1(3), 1-14. 
Hartmann, H. (1980). Un matrimonio mal avenido: hacia una unión más progresiva entre marxismo y feminismo. Zona Abierta, 24, 85-113.

Hidalgo, H. y Rodríguez, N. (2014). El valor simbólico de la prostitución: un análisis a partir de la mirada de las mujeres que la practican y la sociedad que las observa, en la provincia de Puntarenas. Tesis para optar por el grado de licenciatura en Trabajo Social. San Ramón: Costa Rica.

Izquierdo, M. (1998). El malestar en la desigualdad. Madrid: Cátedra.

Jager, M.; López, L. y Vargas, G. (2005). Material de apoyo. Sensibilización en No discriminación por ninguna razón. San José: Poder Judicial.

Lerner, G. (1986). El origen del patriarcado. La creación del patriarcado. Barcelona: Editorial crítica.

Ley orgánica sobre el derecho de las mujeres a una vida libre de violencia. (2007). Ley orgánica sobre el derecho de las mujeres a una vida libre de violencia. Asamblea Nacional de la República Bolivariana de Venezuela.

Maffia, D. (2007). Epistemología feminista: La subversión semiótica de las mujeres en la ciencia. Revista venezolana de estudios de la mujer, 12, 63-98.

Mejía, J. (2004). Sobre la investigación cualitativa. Nuevos conceptos y campos de desarrollo. Revista Investigaciones Sociales, 13(8), 277-299.

Paolini, C. (2011). Violencia perinatal, una nueva conceptualización. Buenos Aires: Universidad de Buenos Aires.

Pelletier, P. (2014). La "discriminación estructural" en la evolución jurisprudencial de la Corte Interamericana de Derechos Humanos. Revista IIDH, 60, 205-215.

Sánchez, S. (2015). La violencia obstétrica desde los aportes de la crítica feminista y la biopolítica. Dilemata, 7(18), 93-111.

Tasies, E. (2015). Actores políticos y actores sociales en el siglo XXI. San José: Universidad de Costa Rica.

Vasilachis, I. (2006). Investigación cualitativa. Barcelona: Gedisa Editorial. 